\title{
Direct experimental evidence of longitudinal and transverse mode hybridization and anticrossing in simple model fluids
}

\author{
Egor V. Yakovlev, ${ }^{1}$ Nikita P. Kryuchkov, ${ }^{1}$ Pavel V. Ovcharov, ${ }^{1}$ Andrei V. Sapelkin, ${ }^{2}$ Vadim V. Brazhkin, ${ }^{3}$ and \\ Stanislav O. Yurchenko ${ }^{1, \text { a) }}$ \\ 1) Bauman Moscow State Technical University, 2nd Baumanskaya street 5, 105005 Moscow, \\ Russia \\ ${ }^{2)}$ School of Physics and Astronomy, Queen Mary University of London, London E1 4NS, \\ England \\ ${ }^{3)}$ Institute for High Pressure Physics RAS, Kaluzhskoe shosse, 14, Troitsk, Moscow, \\ 108840 Russia
}

(Dated: 25 January 2020)

A significant number of key properties of condensed matter are determined by the spectra of elementary excitations and, in particular - collective vibrations. However, behaviour and description of collective modes in disordered media (e.g. liquids and glasses) remains a challenging area of modern condensed matter science. Recently, anticrossing between longitudinal and transverse modes was predicted theoretically and observed in molecular dynamics simulations, but this fundamental phenomenon has never been observed experimentally. Here, we demonstrate the mode anticrossing in a simple Yukawa fluid constructed from charged microparticles in weakly-ionized gas. Theory, simulations, and experiments show clear evidence of mode anticrossing that is accompanied by mode hybridization and strong redistribution of the excitation spectra. Our results provide significant advance in understanding excitations of fluids, opening new prospectives for studies of dynamics, thermodynamics, and transport phenomena in a wide variety of systems from noble gas fluids and metallic melts to strongly coupled plasmas, molecular, and complex fluids.

Due to ubiquitous presence of fluids, from live systems to materials technologies, understanding of their collective dynamics is important for condensed matter, chemical physics, physical chemistry, physics of plasmas, soft matter, and materials science. In crystals, strongly interacting particles move with small amplitudes around their equilibrium positions in a periodic lattice, and the spectra of longitudinal and transverse excitations can be calculated with Born-von Karman method (for known interaction potential)! 1 In glasses, periodicity of lattice is broken, but the magnitude of particle motions is still small and the diffusion is suppressed. This lack of periodicity results in significant theoretical, computational and experimental challenges ${ }^{2}$ As a consequence, collective excitation in glasses has been an area of intense research over the last several decades with a number of important outstanding problems still to be addressed $\left[\begin{array}{l}3-7 \\ -7\end{array}\right.$ Crucially, properties of glasses depend significantly of the structure and dynamics of liquids they obtained from, while understanding the collective excitations in liquids is even more challenging than in the case of glasses.

To address these challenges, we have recently proposed ${ }^{8[9}$ an approach to the treatment of collective excitations in fluids in the framework of pseudoharmonic approximation. We showed that the Hamiltonian of the system can be written in terms of transverse and longitudinal components, with a mode coupling term related to excitation mixing, damping, and dissipation. We found that the coupling term results in mode repulsion leading to a frequency gap in the excita-

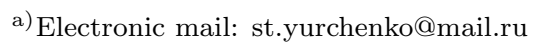

tion spectrum - mode anticrossing. Due to structural disorder, excitations with large wavenumbers are subjected to intense scattering. That leads to their mutual mixing and results in their interaction over the range of wavenumbers, where the longitudinal and transverse modes are typically crossed. As a result, the modes repel resulting in a frequency gap, being accompanied by strong redistribution and hybridization of the spectra near the mode crossing region. Anticrossing is a generic phenomenon, inherent for systems of different nature, including crystals ${ }^{10} 17$ mechanical and electromagnetic curcuits,, 181 metamaterials and phononic crystals, $, 22,24$ polaritonic systems, ${ }^{21 \mid 25}$ solids with defects and thermoelectric crystals with guest atoms, $\frac{1126 \mid 27}{20 p t i c a l ~ a n d ~ l o n-~}$ gitudinal modes in fluids. ${ }^{28] 30}$ However the mode anticrossing in simple fluids has never been experimentally observed or reported.

In this paper, we report for the first time on mode anticrossing in particle-resolved experimental study in a model fluid. This is done by utilizing a system of interacting micro particles with tunable interactions - an equilibrated Yukawa fluid - realized in a dusty plasma system. We present a detailed study of anticrossing of longitudinal and transverse modes in fluids. We find clear evidence of mode anticrossing in the short wavelength region - the region responsible for scattering processes affecting transport phenomena (e.g., viscosity and thermal conductivity). Thus, our findings provide significant advance in understanding excitations in a wide variety of systems, spanning from simple fluids and metallic melts to strongly coupled plasmas and molecular fluids.

Our approach to the experimental investigation of mode anticrossing in fluids consists of two key ingre- 
dients: (i) carefully setting up experimental fluid itself and (ii) data analysis tailored to the problem in the framework of recently developed methodology ${ }^{8}$ (the latter is also described in details in the Supporting Information, $\mathrm{S}^{31}$ ). In order to observe mode anticrossing directly, we performed dedicated experiments with $2 \mathrm{D}$ system of charged microparticles in the same manner as described previously $[22 \underline{36}$ To prepare the fluid, we melted $2 \mathrm{D}$ complex plasma crystal utilising the mode-coupling instability ${ }^{36}$ (see details in $\mathrm{Sl}^{31}$ ), however, the same could be done using laser heating 39440

First, the model fluid system was set up. The experimental video of model fluid used in the present work is shown in Movie S2 $\frac{31}{1 n}$ order to establish that we deal with fluid, we calculated the average mean square displacement (MSDs) of particles

$$
\sigma^{2}(t)=\sum_{\alpha=1}^{N(t)}\left(\mathbf{r}_{\alpha}(t)-\mathbf{r}_{\alpha}(0)\right)^{2} / N(t),
$$

where $\alpha$ denotes the particles being tracked, and $N(t)$ is the number of particles remaining in the area of analysis for the time $t$.

The dimensionless MSDs (1) normalized to the average area per particle in the fluid $V_{0}=0.093 \mathrm{~mm}^{2}, \sigma^{2}(t) / V_{0}$, are shown in Fig. 1(a) for the cases of the crystal before melting and in the fluid. The isotropic pair correlation functions $g(r)$ are shown in Fig. 1(b). One can see that in crystal the particles are localized, strongly correlated spatially, and the diffusion is strongly suppressed, as shown by the blue symbols in Figs. 1(a) and 1(b). On the contrary, as shown for the fluid by the orange symbols, the MSD grows linearly with time (inherently for fluids) and the correlations drop rapidly with the distance between particles. The linear fit $\sigma^{2}(t)=4 D t$ (corresponding to the normal diffusion) is shown in Fig. 11(a) by the solid red line, from where we obtained the diffusion constant $D \simeq 4.8 \times 10^{-2} \mathrm{~mm}^{2} / \mathrm{s}$ and the characteristic time $\tau_{D}=V_{0} / D \simeq 1.92 \mathrm{~s}$. Note that the (slow) logarithmic divergence becomes significant at long times, $t \gg \tau_{D}$, and, in our case it can be neglected.

To further characterize the fluid used in our experiment, we analyzed the distributions of the particle kinetic energy and Voronoi cell area ${ }^{41}$ The results are shown in Fig. 2, In the steady state, the particle kinetic energy obeys the Maxwell distribution, $p(K) \propto \exp (-K / T)$, where $K$ is in-plane particle kinetic energy. The kinetic temperature is determined by the interplay of energy release (due to plasma wakes) and Epstein damping (due to the friction of particles moving in the neutral gas) $\underline{\underline{42143}}$ The experimental distribution and its theoretical fit are shown in Fig. 2(a) by the yellow histogram and solid red line, respectively. This theoretical fit agrees well with the experimental data and gives a value of $T=18 \mathrm{eV}$ for the kinetic temperature of microparticles in our experiment (the melting temperature is about $T_{m} \simeq 9 \mathrm{eV}$ ). The inset of Fig. 2(a) shows the snapshot of the plasma fluid under study (see Movie S231). Note that the "damp-
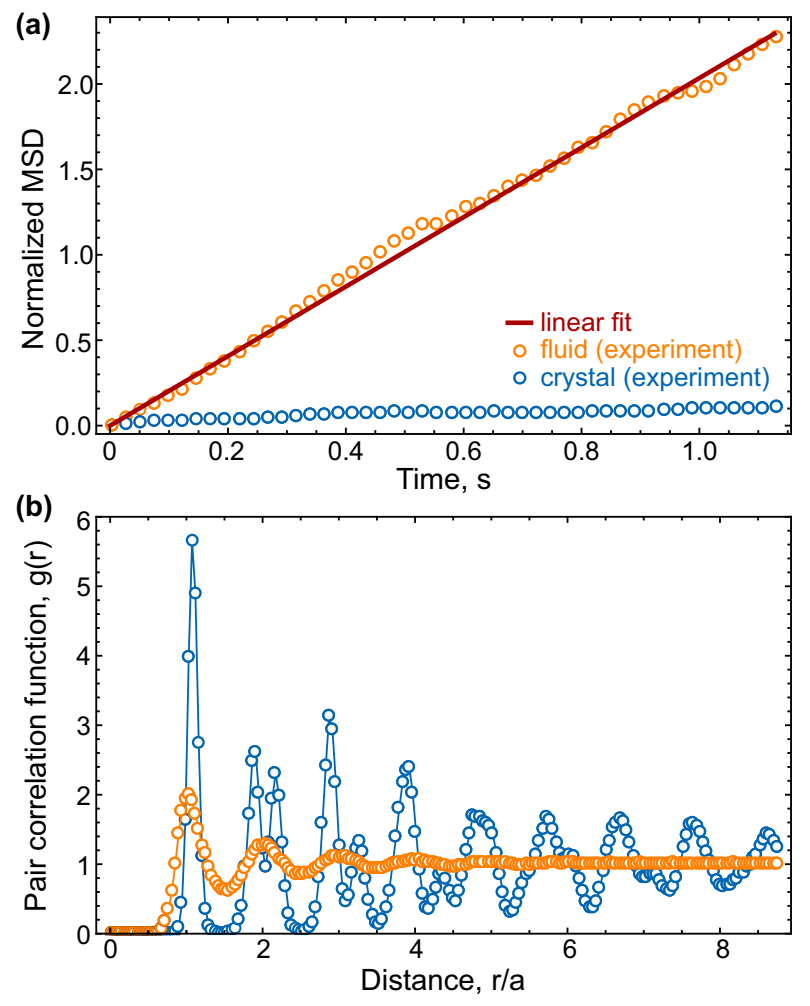

FIG. 1. Diffusion and pair correlations in the experimentally studied monolayer fluid: (a) Experimental dependencies of the normalized MSDs, $\sigma^{2}(t) / V_{0}$, of time for the crystal and fluid shown by the blue and orange symbols (see Movies S1 and S2 ${ }^{31}$ ). Solid red line is linear fit $\sigma^{2}(t)=4 D t$ with the diffusivity constant $D \simeq 4.8 \times 10^{-2} \mathrm{~mm}^{2} / \mathrm{s}$. (b) Experimental $g(r)$-functions of the crystal and fluid. The distances are normalized to the crystalline lattice parameter $a=330 \mu \mathrm{m}$ (see $\left.\mathrm{Sl}^{31}\right)$.

ing strength" parameter $\xi=\nu a \sqrt{m / T}=0.27$ is small (here, $\nu=1.8 \mathrm{~s}^{-1}$ is the Epstein damping rate and $m=6.1 \times 10^{-10} \mathrm{~g}$ is the particle mass ${ }^{31}$ ), that means practically Newtonian (underdamped) dynamics of individual particles ${ }^{[4]}$ On the contrary, since $\tau_{D} \nu=3.46 \gtrsim 1$, the diffusion (expectedly) occurs in Brownian regime.

The distribution of the Voronoi cell area is shown in Fig. 2(b). Equilibrium fluctuations near the average value of the area are determined by the equation of state of the system, since the corresponding probability distribution function $p(V)$ is $\frac{44}{4}$

$$
p(V) \propto \exp \left[\frac{1}{2 T}\left(\frac{\partial P}{\partial V}\right)_{T}\left(V-V_{0}\right)^{2}\right],
$$

where $P$ is the pressure and $V$ is the volume (area in $2 \mathrm{D}$ case) per particle (with the average value $V_{0}=$ $\left.0.093 \mathrm{~mm}^{2}\right)$. The kinetic temperature $T$ and average volume $V_{0}$ used in the distribution (2) were taken from the experiment. Under the assumption that microparticles interact via the Debye-Hückel (Yukawa) potential, the particle charge number $Z=1.25 \times 10^{4}$, screening length $\lambda_{D}=260 \mu \mathrm{m}$, and vertical confinement frequency 

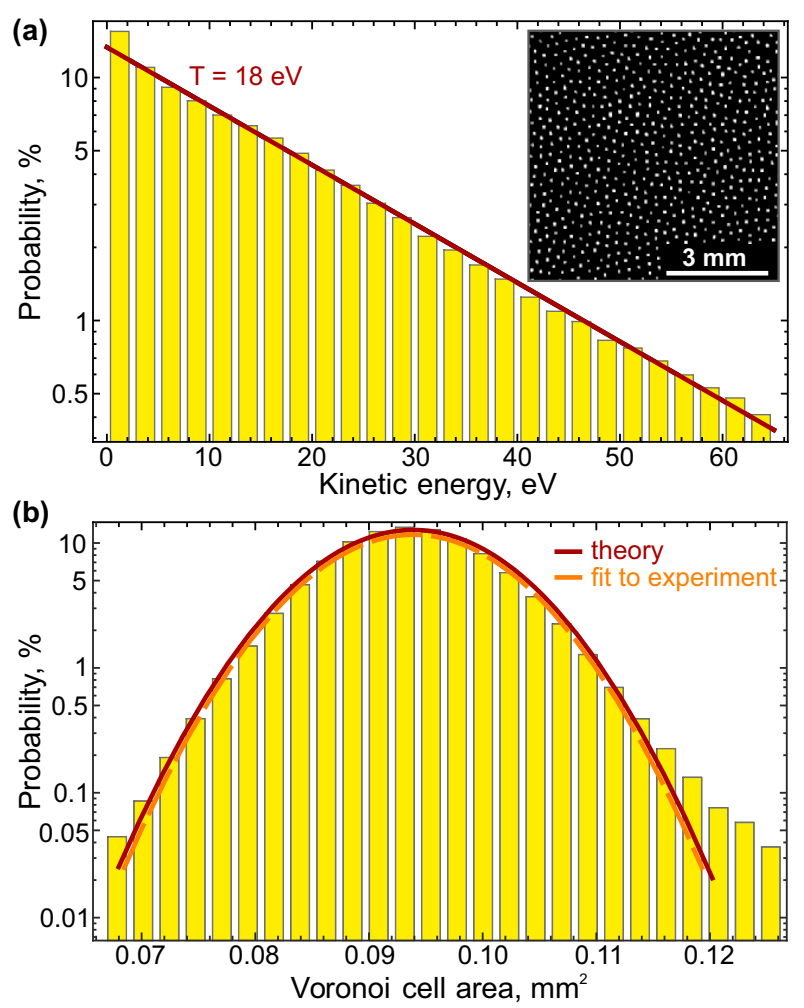

FIG. 2. Characterization of the experimentally studied plasma fluid: (a) Normalized distribution of in-plane kinetic energy of microparticles. The histogram presents experimental results, the solid red line is the Maxwellian distribution with the kinetic temperature of the microparticles $T=18 \mathrm{eV}$ (the crystal melts at $T_{m} \simeq 9 \mathrm{eV}$ ). The inset shows the experimental snapshot of the plasma fluid (see Movie $\mathrm{S} 2^{31}$ ). (b) Experimental distribution of Voronoi cell areas (histogram). The dashed orange line is a gaussian fit. The solid red line is theoretical distribution (2) calculated for the 2D Yukawa system with the particle charge, screening length, kinetic temperature, and density corresponding to experimental values.

$\Omega_{z}=25 \mathrm{~Hz}$ were obtained by fitting the in-plane fluctuation spectra in the plasma crystal to theoretical ones for the Yukawa mode ${ }^{32 \mid 33 / 45]}$ (see details in $\mathrm{Sl}^{31}$ ). Then, for the experimentally measured parameters, we used the theoretical value of $(\partial P / \partial V)_{T}$ for the $2 \mathrm{D}$ Yukawa fluid, $\stackrel{46}{ }$ to make sure our experiment and theory are consistent with each other. Equation (2) can be used to obtain $(\partial P / \partial V)_{T}$ by fitting the experimental $p(V)$ with gaussian, as shown by the dashed orange line in Fig. 2(b).

Data shown in Figs. 2(a) and 2(b) are related to the Maxwell and Boltzmann parts in the Gibbs distribution function ${ }^{44}$ An excellent agreement between experimental and theoretical distributions justifies consideration of our experimental system as a thermalised Yukawa fluid. The screening parameter $\kappa=a_{2 \mathrm{D}} / \lambda_{D} \simeq 0.66$ (where, $a_{2 \mathrm{D}}=\left(V_{0} / \pi\right)^{1 / 2} \simeq 172 \mu \mathrm{m}$ is the $2 \mathrm{D}$ Wigner-Seitz radius), that corresponds to soft repulsive interactions. ${ }^{2]} \mathrm{We}$ analyzed the excitation spectra of in-plane longitudinal and transverse modes in the fluid using the same approaches as we used in Ref. ${ }^{8}$ and explained in $\mathrm{S}^{31}$. Note that the effect of interaction between in- and out-of-plane modes in the fluid was negligible, as evidenced by a long steady-state lifetime of the fluid state $(\sim 1.5 \mathrm{~s})$. Otherwise, fluid $\mathrm{MCl}^{47}$ would have lead to extremely rapid heating of the fluid! $32[33$

The second crucial step is the analysis of the experimental data according to our recently-developed approach ${ }^{8[31}$ In contrast to crystals, fluids do not possess long-range order. As a result, the Brillouin zone based approach to the analysis of phonon dispersion can no longer be employed. Consequently, excitations should be considered for all wavenumbers, from (small- $q$, collective) hydrodynamic to (high- $q$, single-particle) kinetic limit ${ }^{9}$ Comprehensive information about the spectra must include the damping rates, whose values can be comparable with of even larger than the oscillation frequencies and, thus, cannot be neglected.

To obtain excitation spectra in fluids using particle velocities, we should analyze the particle current spectra ${ }^{48}$

$$
C_{\|, \perp}(q, \omega)=\int d t e^{i \omega t} \operatorname{Re}\left\langle\mathbf{j}_{\|, \perp}(\mathbf{q}, t) \mathbf{j}_{\|, \perp}(-\mathbf{q}, 0)\right\rangle,
$$

where $\mathbf{j}(\mathbf{q}, t)=N^{-1} \sum_{s} \mathbf{v}_{s}(t) \exp \left(i \mathbf{q} \mathbf{r}_{s}(t)\right)$ is the velocity current, $N$ is the total number of particles in the system, $\mathbf{v}_{s}(t)=\dot{\mathbf{r}}_{s}(t)$ is the velocity of the $s$-th particle, $\mathbf{j}_{\|}=$ $\mathbf{q}(\mathbf{j} \cdot \mathbf{q}) / q^{2}$ and $\mathbf{j}_{\perp}=\mathbf{j}-\mathbf{q}(\mathbf{j} \cdot \mathbf{q}) / q^{2}$ are the longitudinal and transverse components of the current, and the brackets $\langle\ldots\rangle$ denote the ensemble average.

Assuming that the current-current correlator for longitudinal and transverse excitations is 819

$$
\operatorname{Re}\left\langle\mathbf{j}_{\|, \perp}(\mathbf{q}, t) \mathbf{j}_{\|, \perp}(-\mathbf{q}, 0)\right\rangle \propto e^{-\Gamma_{\|, \perp}(q)|t|} \cos \left(\omega_{\|, \perp}(q) t\right),
$$

we readily obtain from Eqs. (3) and (4) the following Lorentzian fit

$$
\begin{aligned}
& C_{\|, \perp}(q, \omega) \propto \frac{\Gamma_{\|, \perp}(q)}{\left(\omega-\omega_{\|, \perp}(q)\right)^{2}+\Gamma_{\|, \perp}^{2}(q)}+ \\
& +\frac{\Gamma_{\|, \perp}(q)}{\left(\omega+\omega_{\|, \perp}(q)\right)^{2}+\Gamma_{\|, \perp}^{2}(q)},
\end{aligned}
$$

where $\omega_{\|, \perp}(q)$ and $\Gamma_{\|, \perp}(q)$ are the frequency and damping rate of the longitudinal and transverse modes with the wavenumber $q$.

To obtain the frequencies, damping rates, and coupling parameters, we calculated the current spectra using Eq. (3) (for experiment and MD) and then fitted them with Eq. (5) separately or jointly for londitudinal and transverse excitations. ${ }^{831}$ In the experiment, the particle velocities were measured using standard tracking! ${ }^{49 \mid 50}$ In analysis of excitation spectra, the two-oscillator model and the anticrossing-based method provide the same accuracy, but the latter has less free variables (three vs four) 819131

Following the experimental data analysis, the anticrossing of longitudinal and transverse excitations in the 

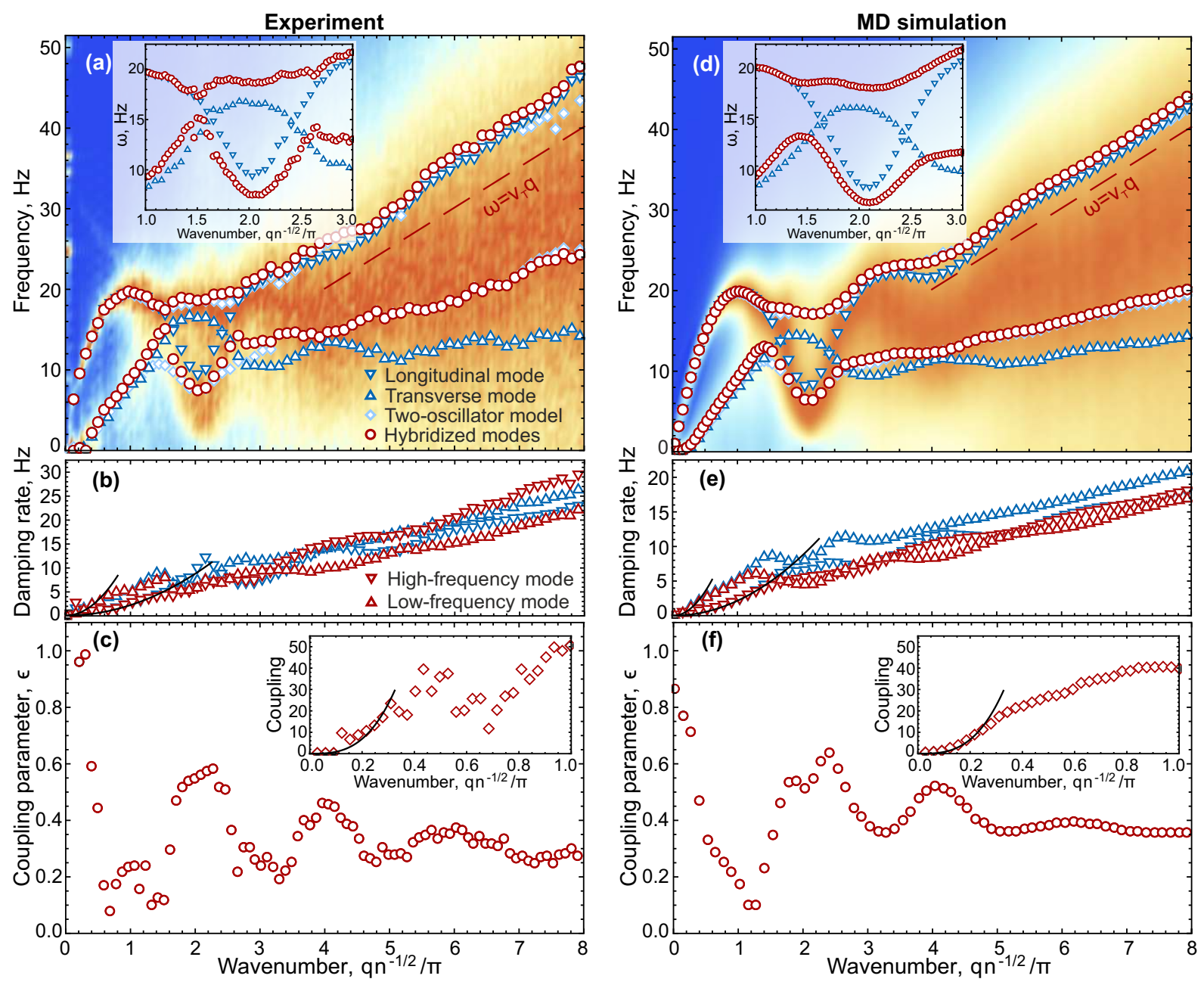

FIG. 3. Mode anticrossing in strongly-coupled plasma fluid in experiment and MD simulation: The spectra, damping rates, and coupling parameters between longitudinal and transverse excitations, obtained from (a)-(c) experiment and (d)-(f) MD simulation. (a), (d) Frequencies $\omega$ of collective modes obtained from separate analysis of longitudinal and transverse fluctuations (blue triangles), two-oscillator model (blue rhombi), and the anticrossing-based method (hybridized modes) shown by red circles. ${ }^{8 / 9131}$ The color map is $C(q, \omega)$ normalized to its maximum at each given $q$. The insets show zooms on the mode anticrossing region. (b), (e) Damping rates $\Gamma$ obtained using separate mode analysis (blue triangles) and the anticrossing-based method (red triangles). Solid black lines are quadratic fits $\Gamma_{\|, \perp} \propto q^{2}$ at small $q$, the same in (b) and (e). (c), (f) The (normalized) coupling parameter $\epsilon$ calculated using anticrossing theory $\sqrt{81}$ and the coupling $\epsilon \Omega_{\|} \Omega_{\perp}$ (shown in the inset). The solid black line is a cubic fit $\epsilon \Omega_{\|} \Omega_{\perp} \propto q^{3}$, the same in (c) and (f).

complex plasma fluid is revealed in Fig. 3, where the results are also compared with MD simulations. The experimentally obtained parameters of the interaction between particles in thus constructed quasi-2D fluid were used in our MD simulations (see details in $\mathrm{Sl}^{31}$ ) and one can see that the experimental and MD results agree very well. Observed differences are caused by better MD statistics and more complicated (compared to the simplified model we used in MD) interactions owing to the plasma wakes in the experiment. Mode anticrossing is seen at $q n^{-1 / 2} / \pi \simeq 1.6$ and 2.4 (in the intermediate range of wavenumbers between collective and single-particle dynamics), where two crossing modes (shown by blue triangles) repel from each other. As a result of the anticross- ing, we observe high- and low-frequency modes of hybridized excitations presented by red circles in Figs. 3(a) and 3 (d), instead of longitudinal and transverse excitations, shown by blue triangles.

In fluids, due to strong damping of excitations, the longitudinal and transverse components of the total current $C(q, \omega)=C_{\|}(q, \omega)+C_{\perp}(q, \omega)$ (shown in Figs. 3(a) and $3(\mathrm{~d}))$ are significantly broadened in $(q, \omega)$ plane 9151,53 The only exception is related to the hydrodynamic limit of longitudinal excitations, since $\omega_{\|} \propto q, \Gamma_{\|} \propto q^{2}$ (see, e.g., Refs. $\frac{54155}{5}$ ), and $\Gamma_{\|} / \omega_{\|} \propto q$ at $q \rightarrow 0$ (where the coupling between the modes vanishes $\frac{831}{131}$ ). The damping rates are presented in Figs. 3(b) and 3(e). The solid black lines show the same (for experiment and 
MD) diffusive damping $\Gamma_{\|, \perp} \propto q^{2}$ at small $q$. The normalized (dimensionless) coupling parameter $\epsilon$ is shown in Figs. 3(c) and 3(f) for experiment and MD simulation. The coupling between modes is described by $\epsilon \Omega_{\|} \Omega_{\perp}$ (here, $\Omega_{\|, \perp}^{2}=\omega_{\|, \perp}^{2}+\Gamma_{\|, \perp}^{2}$ ) and shown in the insets. $\frac{8 \mid 31}{131}$ The solid black line is a cubic fit $\epsilon \Omega_{\|} \Omega_{\perp} \propto q^{3}$, since, at small $q, \Omega_{\|} \simeq \omega_{\|} \propto q$ and $\Omega_{\perp} \simeq \Gamma_{\perp} \propto q^{2}\left(\omega_{\perp}=0\right.$ in the $q$-gap 819131 . One can see a remarkable agreement of experiment and MD results with asymptotic vanishing of the mode damping and coupling at $q n^{-1 / 2} \ll 1$.

At $q n^{-1 / 2} \gg 1$, the collective excitations undergo transition to regime of individual particles movements. The Lorentzian profile (5) was shown in Ref.9 to be unsuitable in this case. Instead, $C(q, \omega)$ profile is being accurately determined by the Maxwell distributions, but for moderate $q n^{-1 / 2} / \pi \lesssim 3$ the fit (5) still works well! ${ }^{9}$ The high-frequency branch of excitations, usually ascribed to longitudinal excitations, approaches the linear asymptote $\omega=v_{T} q$, where $v_{T}=\sqrt{2 T / m}$ is the most probable velocity of the Maxwell distribution ${ }^{9}$, as shown by the dashed red lines in Figs. 3(a) and 3(d).

The peaks of $C_{\|, \perp}(q, \omega)$ are significantly broadened due to large damping. As a result, the sum $C(q, \omega)$ does not drop in the range between the frequencies $\omega_{\|, \perp}$ (as shown in Figs. 3(a) and 3(d)), but the modes are still distinguishable using our fitting procedures. In the regions of mode anticrossing, the excitations have mixed (elliptic) polarization and corresponding branches are separated by hybridization gap in $(q, \omega)$-plane. To confirm this interpretation, we demonstrate in Figs. S2 and $S 3^{31}$ the currents $C_{\|, \perp}(q, \omega)$ (for the data shown in Fig. 3), which slightly drop in the areas of their "interference" (in the vicinity $q n^{-1 / 2} / \pi=(1.0 \ldots 1.7)$ and $\left.\omega=(10 \ldots 15) \mathrm{Hz}\right)$ due to the mode anticrossing, hybridization, and the polarization mixing. Taking into account that $\tau_{D} \simeq 2 \mathrm{~s}$ in our experiments, we conclude that $\omega \tau_{D} \simeq 30 \gg 1$ for the characteristic frequencies of anticrossing. This constitutes a clear proof that the coupling parameter $\epsilon$ can indeed be considered constant and its (slow) fluctuations can be neglected during analysis of the mode anticrossing! 8

Thus, our approach to the data analysis, allows to demonstrate that mode anticrossing results in strong redistribution of excitation spectra, accompanied by the formation of hybridized high- and low-frequency excitations (instead of longitudinal and transverse ones) in the short wavelength region. This suggests that anticrossing approach should be the key element of analysis of spatially-localized rearrangements and collective excitations in atomic and molecular systems since the intermediate range of wavenumbers correspond to the transition from collective to single-particle dynamics? The anticrossing is a generic phenomenon, and we expect the same behavior can be observed in various disordered systems, including simple, molecular, and ionic fluids, liquid metals, and glasses.

In summary, our study provides a clear evidence that mixing of modes in strongly coupled fluids results in ef- fective interaction between longitudinal and transverse excitations leading to mode anticrossing. The observed trends are similar to those reported in Ref. ${ }^{8}$ for fluid noble gasses, which strongly suggests that anticrossing is a generic phenomenon, intrinsic for fluids of different nature and disordered structures in general. Hence, the role of anticrossing in crystallization and vitrification should also be studied in future. We show that the anticrossingbased method for analysis of excitations allows to obtain frequencies and damping rates. Consequently, we believe that detailed analysis of excitations opens up exciting prospects for elaboration of transport phenomena theory and establishing (as yet unknown) relations between individual, collective dynamics, and thermodynamics of fluids. 2156] 65

Furthermore, redistribution of the excitation spectra (as result of their anticrossing), can affect the interaction between longitudinal, transverse, and polarization modes in ionic and polar molecular liquids. We leave these interesting problems for future work.

\section{ACKNOWLEDGMENTS}

The study was supported by the Russian Science Foundation, Grant No. 19-12-00111 (MD data analysis) and Grant No. 17-19-01691 (experiments, theory, MD simulation, and discussion).

\section{SUPPORTING INFORMATION AVAILABLE:}

Details of anticrossing theory of longitudinal and transverse excitations in simple fluids; technical details of experiments, MD simulations, and data analysis; experimental excitation spectra in a $2 \mathrm{D}$ plasma crystal (Fig. S1), longitudinal and transverse components of particle currents spectra in the model fluid (Fig. S2), selected sections of particle currents spectra in Fig. S2 at different wavenumbers (Fig. S3).

\section{AUTHOR CONTRIBUTIONS}

E.V.Y. performed experiments; N.P.K. performed MD simulations; N.P.K., P.V.O., and S.O.Y. processed experimental results and MD simulations; E.V.Y., N.P.K., A.V.S., V.V.B., and S.O.Y. analyzed and discussed the results; S.O.Y. conceived, directed the research, developed theory, and wrote the manuscript. All authors reviewed the manuscript.

\section{ADDITIONAL INFORMATION}

Competing interests: The authors declare no competing interests. 
${ }^{1}$ M. Born and K. Huang, Dynamical Theory of Crystal Lattices (Oxford, 1954).

${ }^{2}$ N. H. March and M. P. Tossi, Introduction to liquid state physics (London: World Scientific Publishing, 2002).

${ }^{3}$ G. N. Greaves, A. L. Greer, R. S. Lakes, and T. Rouxel, Nature Materials 10, 823 (2011)

${ }^{4}$ L. Berthier and G. Biroli, Reviews of Modern Physics 83, 587 (2011)

${ }^{5}$ L. Berthier and J. Kurchan, Nature Physics 9, 310 (2013)

${ }^{6}$ D. Bonn, M. M. Denn, L. Berthier, T. Divoux, and S. Manneville, Reviews of Modern Physics 89, 035005 (2017)

${ }^{7}$ F. H. Stillinger and P. G. Debenedetti, Annual Review of Condensed Matter Physics 4, 263 (2013)

${ }^{\gamma}$ N. P. Kryuchkov, V. V. Brazhkin, and S. O. Yurchenko, The Journal of Physical Chemistry Letters 10, 4470 (2019)

${ }^{9}$ N. P. Kryuchkov, L. A. Mistryukova, V. V. Brazhkin, and S. O. Yurchenko, Scientific Reports 9, 10483 (2019)

${ }^{10} \mathrm{M}$. T. Dove, Introduction to Lattice Dynamics (Cambridge University Press, 1993).

${ }^{11}$ M. Christensen, A. B. Abrahamsen, N. B. Christensen, F. Juranyi, N. H. Andersen, K. Lefmann, J. Andreasson, C. R. H. Bahl, and B. B. Iversen, Nature Materials 7, 811 (2008)

${ }^{12}$ M. Beekman, D. T. Morelli, and G. S. Nolas, Nature Materials 14, $1182(2015)$

${ }^{13}$ C. W. Li, J. Hong, A. F. May, D. Bansal, S. Chi, T. Hong, G. Ehlers, and O. Delaire, Nature Physics 11, 1063 (2015)

${ }^{14}$ E. S. Toberer, A. Zevalkink, and G. J. Snyder, Journal of Materials Chemistry 21, 15843 (2011)

${ }^{10}$ D. J. Voneshen, K. Refson, E. Borissenko, M. Krisch, A. Bosak, A. Piovano, E. Cemal, M. Enderle, M. J. Gutmann, M. Hoesch, M. Roger, L. Gannon, A. T. Boothroyd, S. Uthayakumar, D. G. Porter, and J. P. Goff, Nature Materials 12, 1028 (2013)

${ }^{16}$ T. Kumagai, K. Nakamura, S. Yamada, and T. Ohnuma, The Journal of Chemical Physics 145, 064702 (2016)

${ }^{17}$ B. Li, H. Wang, Y. Kawakita, Q. Zhang, M. Feygenson, H. L. Yu, D. Wu, K. Ohara, T. Kikuchi, K. Shibata, T. Yamada, X. K. Ning, Y. Chen, J. Q. He, D. Vaknin, R. Q. Wu, K. Nakajima, and M. G. Kanatzidis, Nature Materials 17, 226 (2018)

${ }^{18}$ A. Ishimaru, Electromagnetic Wave Propagation, Radiation, and Scattering (John Wiley \& Sons, Inc., 2017).

${ }^{19} \mathrm{~A}$. Hardy and W. Streifer, IEEE Journal of Quantum Electronics 22, 528 (1986)

${ }^{20}$ L. Tsang and S.-L. Chuang, Journal of Lightwave Technology 6 , $304(1988)$

${ }^{21}$ A. F. Kockum, A. Miranowicz, S. D. Liberato, S. Savasta, and F. Nori, Nature Reviews Physics 1, 19 (2019)

${ }^{22}$ B. L. Davis and M. I. Hussein, Physical Review Letters 112, 055505 (2014).

${ }^{23}$ N. Zen, T. A. Puurtinen, T. J. Isotalo, S. Chaudhuri, and I. J. Maasilta, Nature Communications 5, 3435 (2014)

${ }^{24}$ B. Graczykowski, M. Sledzinska, F. Alzina, J. Gomis-Bresco, J. S. Reparaz, M. R. Wagner, and C. M. S. Torres, Physical Review B 91, $075414(2015)$

${ }^{25}$ E. Togan, H.-T. Lim, S. Faelt, W. Wegscheider, and A. Imamoglu, Physical Review Letters 121, 227402 (2018)

${ }^{26}$ M. Klinger and A. Kosevich, Physics Letters A 280, 365 (2001)

${ }^{27}$ M. Baggioli, B. Cui, and A. Zaccone, Physical Review B 100, 220201 (2019)

${ }^{2 \gamma}$ C. Petrillo, F. Sacchetti, B. Dorner, and J.-B. Suck, Physical Review E 62, 3611 (2000)

${ }^{29}$ F. Sacchetti, J.-B. Suck, C. Petrillo, and B. Dorner, Physical Review E 69, 061203 (2004)

${ }^{30}$ M. Inui, A. Q. R. Baron, Y. Kajihara, K. Matsuda, S. Hosokawa, K. Kimura, Y. Tsuchiya, F. Shimojo, M. Yao, S. Tsutsui, D. Ishikawa, and K. Tamura, Journal of Physics: Condensed Matter 30, 28LT02 (2018)

${ }^{31}$ See Supporting Information (URL).

${ }^{32}$ S. O. Yurchenko, E. V. Yakovlev, L. Couedel, N. P. Kryuchkov, A. M. Lipaev, V. N. Naumkin, A. Y. Kislov, P. V. Ovcharov, K. I. Zaytsev, E. V. Vorob'ev, G. E. Morfill, and A. V. Ivlev,
Physical Review E 96, 043201 (2017).

${ }^{33}$ N. P. Kryuchkov, E. V. Yakovlev, E. A. Gorbunov, L. Couedel, A. M. Lipaev, and S. O. Yurchenko, Physical Review Letters 121, 075003 (2018)

${ }^{34}$ E. V. Yakovlev, N. P. Kryuchkov, P. V. Ovcharov, K. Pitiot, A. V. Sapelkin, and S. O. Yurchenko, Physical Review E 100, $023203(2019)$

${ }^{35}$ E. V. Yakovlev, M. Chaudhuri, N. P. Kryuchkov, P. V. Ovcharov, A. V. Sapelkin, and S. O. Yurchenko, The Journal of Chemical Physics 151, 114502 (2019)

${ }^{36}$ L. Couedel, V. Nosenko, S. Zhdanov, A. V. Ivlev, I. Laut, E. V. Yakovlev, N. P. Kryuchkov, P. V. Ovcharov, A. M. Lipaev, and S. O. Yurchenko, Physics-Uspekhi 62, 1000 (2019)

${ }^{37}$ A. V. Ivlev and G. Morfill, Physical Review E 63, 016409 (2000)

${ }^{38}$ L. Couedel, V. Nosenko, A. V. Ivlev, S. K. Zhdanov, H. M. Thomas, and G. E. Morfill, Physical Review Letters 104, 195001 (2010)

${ }^{39}$ V. Nosenko, J. Goree, and A. Piel, Physics of Plasmas 13, 032106 (2006)

${ }^{40}$ V. Nosenko, S. Zhdanov, A. V. Ivlev, G. Morfill, J. Goree, and A. Piel, Physical Review Letters 100, 025003 (2008)

${ }^{41}$ P. V. Ovcharov, N. P. Kryuchkov, K. I. Zaytsev, and S. O. Yurchenko, The Journal of Physical Chemistry C 121, 26860 (2017)

${ }^{42}$ A. Ivlev, H. Löwen, G. Morfill, and C. P. Royall, Complex Plasmas and Colloidal Dispersions: Particle-Resolved Studies of Classical Liquids and Solids (Word Scientific, Singapore, 2012).

${ }^{43}$ N. P. Kryuchkov, A. V. Ivlev, and S. O. Yurchenko, Soft Matter 14, $9720(2018)$

${ }^{44}$ L. D. Landau and E. M. Lifshitz, Statistical Physics. Vol. 5 (3rd ed.) (Elsevier, Oxford, 1980).

${ }^{45}$ S. K. Zhdanov, A. V. Ivlev, and G. E. Morfill, Physics of Plasmas 16, $083706(2009)$

${ }^{46}$ N. P. Kryuchkov, S. A. Khrapak, and S. O. Yurchenko, The Journal of Chemical Physics 146, 134702 (2017)

${ }^{4 /}$ A. Ivlev, S. Zhdanov, M. Lampe, and G. Morfill, Physical Review Letters 113, 135002 (2014)

${ }^{48} \mathrm{~J}$.-P. Hansen and I. R. MacDonald, Theory of simple liquids (London: Academic, 2006).

${ }^{49}$ S. S. Rogers, T. A. Waigh, X. Zhao, and J. R. Lu, Physical Biology 4, 220 (2007)

${ }^{50}$ Y. Feng, J. Goree, and B. Liu, Review of Scientific Instruments 78, $053704(2007)$

${ }^{{ }^{1}}$ S. A. Khrapak, N. P. Kryuchkov, L. A. Mistryukova, A. G. Khrapak, and S. O. Yurchenko, The Journal of Chemical Physics 149, $134114(2018)$

${ }^{52}$ S. A. Khrapak, N. P. Kryuchkov, and S. O. Yurchenko, Physical Review E 97, 022616 (2018)

${ }^{53}$ S. A. Khrapak, A. G. Khrapak, N. P. Kryuchkov, and S. O. Yurchenko, The Journal of Chemical Physics 150, 104503 (2019)

${ }^{54}$ M. Baggioli and A. Zaccone, Physical Review Research 1, 012010 (2019)

${ }^{55}$ M. Baggioli and A. Zaccone, Physical Review Letters 122, 145501 (2019)

${ }^{56} \mathrm{~K}$. Trachenko and V. V. Brazhkin, Reports on Progress in Physics 79, $016502(2015)$

${ }^{5}$ V. V. Brazhkin, Physics-Uspekhi 60, 954 (2017)

${ }^{58}$ V. V. Chaban and O. V. Prezhdo, The Journal of Physical Chemistry B 120, $4302(2016)$

${ }^{5 y}$ V. V. Chaban and O. V. Prezhdo, The Journal of Physical Chemistry Letters 3, 1657 (2012).

${ }^{{ }^{\circ}}$ T. Bryk, A. Huerta, V. Hordiichuk, and A. D. Trokhymchuk, The Journal of Chemical Physics 147, 064509 (2017)

${ }^{61}$ S. Khrapak, B. Klumov, and L. Couëdel, Scientific Reports 7, 7985 (2017)

${ }^{02}$ V. V. Chaban and O. V. Prezhdo, The Journal of Physical Chemistry Letters 5, 1973 (2014).

${ }^{63}$ C. Yang, M. T. Dove, V. V. Brazhkin, and K. Trachenko, Phys. Rev. Lett. 118, 215502 (2017) 
${ }^{64}$ S. A. Khrapak, N. P. Kryuchkov, S. O. Yurchenko, and H. M. Thomas, The Journal of Chemical Physics 142, 194903 (2015).

${ }^{65}$ L. Wang, C. Yang, M. T. Dove, A. V. Mokshin, V. V. Brazhkin, and K. Trachenko, Scientific Reports 9, 755 (2019) 